Ann. Biol. anim. Bioch. Biophys., I971, 11 (3), 505-510.

\title{
INFLUENCE DE LA TENEUR EN ISOLEUCINE DU REGIME SUR LA CROISSANCE ET LA TENEUR EN ISOLEUCINE LIBRE DU SANG ET DU MUSCLE DU RAT
}

\author{
M. PAWLAK et R. PION \\ Station d'Étude des Métabolismes, \\ Centre de Recherches de Clermont-Ferrand, I. N. R. A., \\ 63 - Saint-Genes-Champanelle
}

Des études effectuées précédemment sur rats (Pawlak et Pion, I968 $a, b$ ) ont montré que les teneurs en lysine et thréonine libres du sang et du muscle sont en relation directe avec les teneurs en ces acides aminés des régimes. Ces expériences ont permis de déterminer les concentrations en lysine et en thréonine alimentaires à partir desquelles ces acides aminés s'accumulent de façon importante dans les tissus. Nous avons cherché à étendre cette méthode de mesure de la satisfaction du besoin en un acide aminé à un autre acide aminé indispensable, l'isoleucine, dont le métabolisme est très différent de celui de la lysine et de la thréonine (MEISTER, r965).

Seize lots de 8 rats répartis en 4 expériences successives reçoivent à partir du poids de IIo $\mathrm{g}$ pendant une durée de $\mathrm{I}_{4}$ à $\mathrm{I}_{7}$ jours, des régimes semi-synthétiques (tabl. I) à base d'amidon, équilibrés en minéraux et vitamines et dans lesquels l'apport azoté est assuré exclusivement par des acides aminés libres, en proportions telles que les besoins sont satisfaits, sauf en ce qui concerne l'isoleucine, dont la teneur dans les rations varie de 2 à $6 \mathrm{~g} / \mathrm{kg}$ de MS, soit de 36 à I ro p. roo de l'estimation du besoin. Les valeurs retenues pour les besoins en acides aminés indispensables sont celles établies par RANHOTRA et ConNOR-Johnson en I965, sauf dans le cas de la lysine et de la thréonine pour lesquelles nous avons utilisé les résultats de nos expériences précédentes (PAwlak et Pion, Ig68 $a, b$ ).

Les gains de poids vif des animaux et les quantités d'aliments ingérées sont mesurés. A la fin de la période expérimentale les animaux sont sacrifiés après anesthésie à l'éther; le sang total et les muscles des membres postérieurs sont prélevés et leurs teneurs en acides aminés libres sont déterminées après extraction (PAWLAK et Pron, r968 a) par chromatographie sur colonnes au moyen d'un analyseur automatique.

Les résultats suivants ont été obtenus (tabl. 2).

Expérience I : les teneurs en isoleucine des 2 régimes correspondent respectivement à 36 et à $54 \mathrm{p}$. roo du besoin. Les gains de poids et les quantités d'aliment consommées sont faibles surtout dans le premier cas. Les concentrations en isoleucine sanguine des animaux des 2 lots sont voisines, alors que les concentrations musculaires diffèrent de $27 \mathrm{p}$. roo. 


\section{TABLEAU I}

Composition du régime à base d'acides aminés industriels (g)

Taux protéique $(\mathrm{N} \times 6,25)=13 \mathrm{p} .100$

Énergie brute $=4500 \mathrm{kcal} / \mathrm{kg}$ de MS

\begin{tabular}{|c|c|c|c|c|}
\hline L-Thréonine & 4,5 & I,-Phénylalanine & & \\
\hline Acide L-aspartique ... & 5,9 & L-Lysine $\mathrm{HCl} \ldots \ldots$ & $\begin{aligned} 5,7 \\
11\end{aligned}$ & \\
\hline L-Sérine. . . . . . . & 5,8 & L-Tryptophane . . . . . . & 1,7 & \\
\hline Glutamine ......... & 20,5 & L-Histidine $\mathrm{HCl} \mathrm{H}_{2} \mathrm{O}$. & 3,8 & \\
\hline Acide I-glutamique .. & 20,5 & L-Arginine $\mathrm{HCl} \ldots \ldots$ & 7 & \\
\hline L-Proline $\ldots \ldots \ldots \ldots$ & 12 & Agar-Agar $\ldots \ldots \ldots$ & 20 & \\
\hline Glycine $\ldots \ldots \ldots$ & 4,8 & Mélange minéral. . & 60 & \\
\hline L-Alanine $\ldots \ldots \ldots$ & 4,4 & Mélange vitaminique.. & 20 & \\
\hline L-Valine. . . . . . . & 5,9 & Amidon $\ldots \ldots \ldots \ldots$ & 699,25 & \\
\hline L-Cystine ..... & 3 & Huile d'arachide & 64 & \\
\hline DLMéthionine. . & 3,2 & * Biotine ......... & 5 & n! \\
\hline L-Isoleucine . . . & 2,0 à 6,0 & **Chlorure de choline. & 6 & - \\
\hline L-Leucine $\ldots \ldots$ & 8,3 & Soude $2 \mathrm{~N} \ldots \ldots \ldots$ & 40 & - \\
\hline L-Tyrosine $\ldots$. & 3,8 & Eau ............ & 550 & - \\
\hline
\end{tabular}

* Biotine : solution à $40 \mathrm{mg} / \mathrm{litre}$

** Chlorure de choline : solution à $200 \mathrm{~g} /$ litre

\section{TABLEAU 2}

Croissance et consommation des animaux : teneurs en isoleucine libre des tissus sanguins et musculaives

\begin{tabular}{|c|c|c|c|c|c|c|}
\hline \multirow{2}{*}{$\begin{array}{l}\text { Isoleucine } \\
\text { du régime } \\
\text { g/kg M. S }\end{array}$} & \multirow{2}{*}{$\begin{array}{l}\text { Isoleucine } \\
\text { ingérée } \mathrm{mg} / \mathrm{j}\end{array}$} & \multirow{2}{*}{$\begin{array}{l}\text { Gain de poids } \\
\text { moyen jour- } \\
\text { nalier g/jour }\end{array}$} & \multirow{2}{*}{$\begin{array}{c}\text { Consomma- } \\
\text { tion d'aliment } \\
\text { g M.S. par j }\end{array}$} & \multirow{2}{*}{$\begin{array}{c}\begin{array}{c}\text { Gain poids } \\
\times 100\end{array} \\
\begin{array}{c}\text { Consomma- } \\
\text { tion }\end{array}\end{array}$} & \multicolumn{2}{|c|}{ Isoleucine des tissus } \\
\hline & & & & & $\begin{array}{l}\text { Sang } \\
\mathrm{mg} / 100 \mathrm{~g}\end{array}$ & $\begin{array}{c}\text { Muscle } \\
\mathrm{mg} / 100 \mathrm{~g}\end{array}$ \\
\hline $\begin{array}{r}\text { Exp. } \quad \begin{array}{r}2,0 \\
3,0\end{array}\end{array}$ & $\begin{array}{l}17,0 \\
36,0\end{array}$ & $\begin{array}{l}0,4 \\
3,0\end{array}$ & $\begin{array}{r}8,7 \\
11,9\end{array}$ & $\begin{array}{r}4,6 \\
25,6\end{array}$ & $\begin{array}{l}0,53 \\
0,47\end{array}$ & $\begin{array}{l}085 \\
1,08\end{array}$ \\
\hline $\begin{array}{rr}\text { Exp. II } 2,25 \\
2,50 \\
2,75 \\
3,25 \\
3,50\end{array}$ & $\begin{array}{l}22,0 \\
24,0 \\
30 \\
36,0 \\
45,0\end{array}$ & $\begin{array}{l}0,8 \\
1,3 \\
2,2 \\
2,8 \\
4,0\end{array}$ & $\begin{array}{r}9,9 \\
9,7 \\
10,8 \\
11,2 \\
12,9\end{array}$ & $\begin{array}{r}8,1 \\
13,6 \\
20,3 \\
24,6 \\
31,6\end{array}$ & $\begin{array}{l}0,37 \\
0,35 \\
\overline{0,75} \\
0,49\end{array}$ & $\begin{array}{l}0,40 \\
0,58 \\
0,63 \\
0,79 \\
0,72\end{array}$ \\
\hline $\begin{aligned} \text { Exp. III } 4,0 \\
4,25 \\
4,50\end{aligned}$ & $\begin{array}{l}57,0 \\
51,0 \\
64,0\end{array}$ & $\begin{array}{l}4,5 \\
3,85 \\
4,60\end{array}$ & $\begin{array}{l}14,1 \\
12,1 \\
14,3\end{array}$ & $\begin{array}{l}31,7 \\
31,7 \\
32,2\end{array}$ & $\begin{array}{l}0,43 \\
0,60 \\
0,65\end{array}$ & $\begin{array}{l}0,52 \\
048 \\
0,60\end{array}$ \\
\hline $\begin{aligned} & \text { Exp. IV } 4,75 \\
& 5,0 \\
& 5,25 \\
& 5,50 \\
& 5,75 \\
& 6,0\end{aligned}$ & $\begin{array}{l}62,0 \\
75,0 \\
76,0 \\
77,0 \\
84,0 \\
85,0\end{array}$ & $\begin{array}{l}4,25 \\
5,8 \\
5,6 \\
5,45 \\
6,20 \\
5,70\end{array}$ & $\begin{array}{l}13,0 \\
15,0 \\
14,5 \\
14,0 \\
14,6 \\
14,2\end{array}$ & $\begin{array}{l}32,9 \\
38,6 \\
38,7 \\
39,0 \\
42,0 \\
40,5\end{array}$ & $\begin{array}{l}0,79 \\
0,80 \\
0,75 \\
0,65 \\
085 \\
0,79\end{array}$ & $\begin{array}{l}082 \\
0,70 \\
0,87 \\
0,77 \\
0,94 \\
0,94\end{array}$ \\
\hline
\end{tabular}


Expérience II : les gains de poids moyens augmentent régulièrement de 0,8 à $4,0 \mathrm{~g} / \mathrm{j}$ lorsque les teneurs en isoleucine des régimes varient de 40 à $63 \mathrm{p}$. roo du besoin. Les croissances les plus élevées sont obtenues avec les animaux dont la consommation d'aliment est supérieure de $3 \circ \mathrm{p}$. Ioo à celle des rats dont le régime est le plus carencé en isoleucine. Les teneurs en isoleucine libre du sang et du muscle augmentent généralement avec l'apport d'isoleucine du régime, les teneurs du sang restent légèrement inférieures à celles du muscle. Les teneurs extrêmes dans le tissu musculaire sont dans le rapport $180 /$ I00.

Expérience III : les teneurs en isoleucine des régimes varient de 72 à $82 \mathrm{p}$. Ioo du besoin, les gains de poids et les consommations observées sont plus élevés que précédemment cependant que les teneurs en isoleucine libre des tissus sont relativement faibles, en particulier dans le cas du muscle.

Expérience IV : les apports d'isoleucine des régimes s'échelonnent de 90 à I Io p. Ioo du besoin admis. La croissance obtenue lorsque l'aliment contient au moins $5 \mathrm{~g}$ d'isoleucine par $\mathrm{kg}$ MS est voisine de celles qui sont habituellement observées avec des régimes équilibrés ; un régime dont la teneur en isoleucine est inférieure de ro p. 100 au besoin généralement admis permet donc une croissance maximum. Des apports d'isoleucine supérieurs à cette teneur (jusqu'à I Io p. Ioo du besoin) ne modifient pas le gain de poids des animaux et affectent peu la consommation d'aliment. Les teneurs en isoleucine libre des tissus sont plus élevées que celles de l'expérience III, mais diffèrent peu d'un lot à l'autre.

Les croissances des animaux qui ont consommé les régimes les plus fortement carencés en isoleucine sont beaucoup plus faibles que celles qui ont été obtenues avec des animaux dont la ration, à base de blé, comportait un déficit en lysine du même ordre de grandeur (PAwlak et PION, 1968 a) ; elles sont en revanche analogues à celles qui ont été observées dans le cas de rations à base d'acides aminés libres carencées en thréonine (données non publiées). Ces croissances plus faibles sont liées en partie à une diminution importante des quantités ingérées.

Dans l'ensemble de ces expériences, (tabl, 2) la quantité ingérée, le gain de poids journalier et le rapport gain de poids/consommation d'aliments sont d'autant plus élevés que le régime est plus riche en isoleucine; cependant, ces paramètres ne semblent plus augmenter quand le régime contient au moins $5 \mathrm{~g}$ d'isoleucine/ $\mathrm{kg}$ de MS, ce qui correspond à une ingestion iournalière de $75 \mathrm{mg}$ de cet acide aminé.

Contrairement aux résultats obtenus pour la lysine et la thréonine qui s'accumulent dans les tissus à partir de la satisfaction du besoin, l'isoleucine reste en concentration faible dans les deux tissus étudiés. La lysine et la thréonine libre (fig. I et fig. 2), du fait de leur non utilisation pour la synthèse protéique entraînée par le déficit important en isoleucine, s'accumulent dans le tissu musculaire des animaux qui ont consommé les régimes les plus déficients en isoleucine. La teneur en histidine libre du muscle augmente avec les quantités d'isoleucine ingérées (fig. 3) et avec la quantité d'aliment consommé. Les teneurs en valine et en isoleucine du tissu musculaire (fig. 4) ne sont pas sensiblement modifiées par les différences de teneur en isoleucine des régimes. Les teneurs de l'ensemble des acides aminés indispensables du tissu musculaire diminuent lorsque les quantités d'isoleucine ingérées (fig. 5) et de matière sèche ingérées (fig. 7) augmentent. L'ensemble des acides aminés non indispensables évoluent en sens inverse (fig. 6) et (fig. 8).

L'absence d'accumulation de l'isoleucine dans le tissu musculaire pourrait s'expliquer par la dégradation rapide de l'isoleucine au niveau des tissus périphériques. Ces résultats sont en accord avec ceux obtenus par RICHARDSON et al. (1965) sur des poulets : pour une augmentation de la teneur en isoleucine des régimes de 7,8 à $15,6 \mathrm{~g} / \mathrm{kg}$ obtenue par une augmentation de la concentration azotée des rations $(N \times 6,25)$ de 15 à $30 \mathrm{p}$. Ioo, ces auteurs n'observent pas d'angmentation de l'isoleucine libre dans le tissu musculaire.

Ces résultats sont également en accord avec ceux obtenus en faisant varier la teneur en isoleucine des régimes de rats de celles des autres acides aminés en augmentant le taux protéique ( 14,0 à 20,5 p. roo) de rations à 

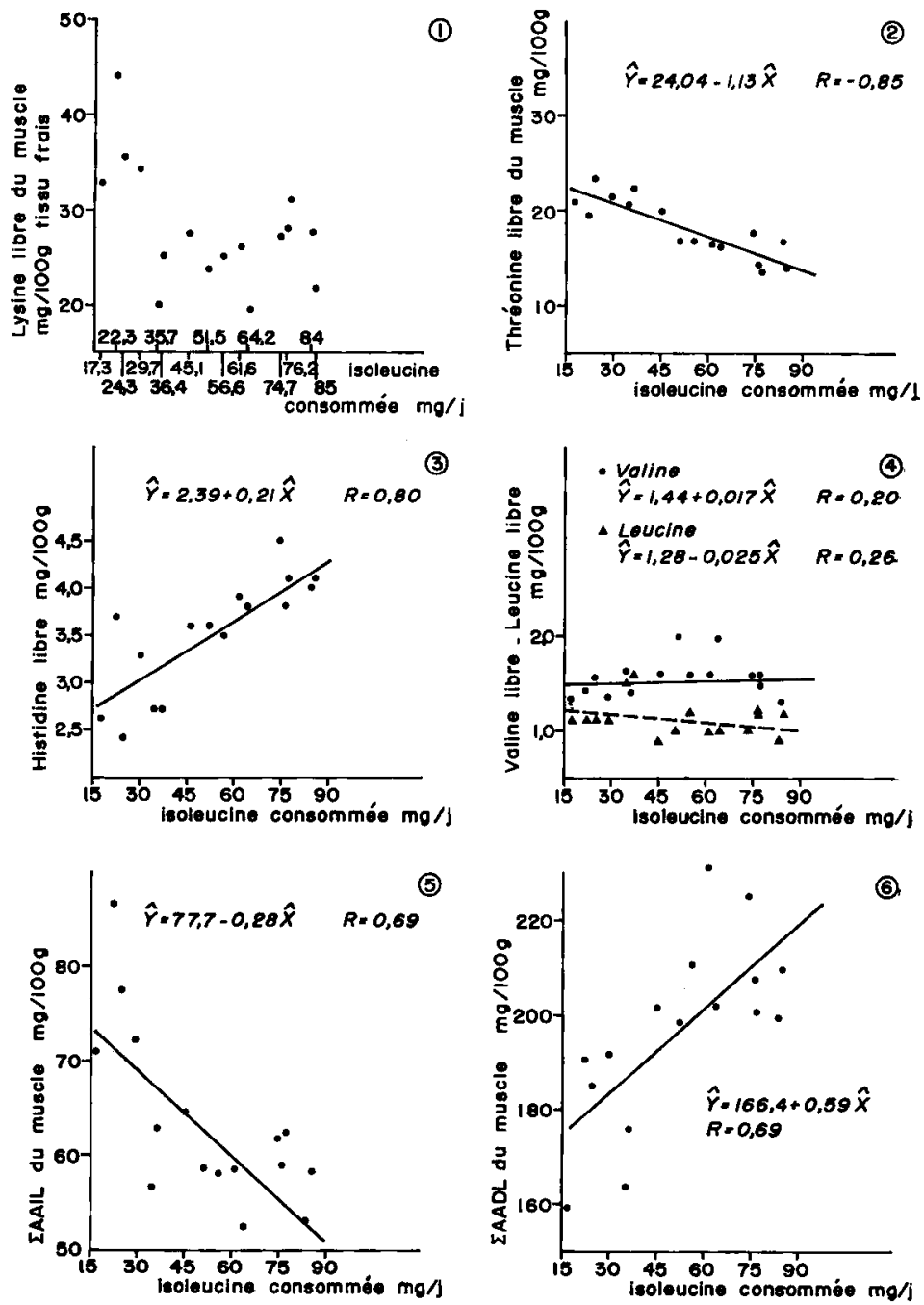

FIG. I. - Teneurs en lysine libre du muscle en fonction de la quantité d'isoleucine ingérée FIG. 2. - Teneurs en thréonine libre du muscle en fonction de la quantité d'isoleucine ingérée FIG. 3. - Teneurs en histidine libre du muscle en fonction de la quantité d'isoleucine ingérée.

FrG. 4. - Teneurs en leucine et valine libre du muscle en fonction de la quantité d'isoleucine ingérée

FIG. 5. - Somme des acides aminés libres indispensables du muscle en fonction de la quantité d'isoleucine ingerée

FIc. 6. - Somme des acides aminés non indispensables libres du muscle en fonction de la quantite d'isoleucine ingérée 

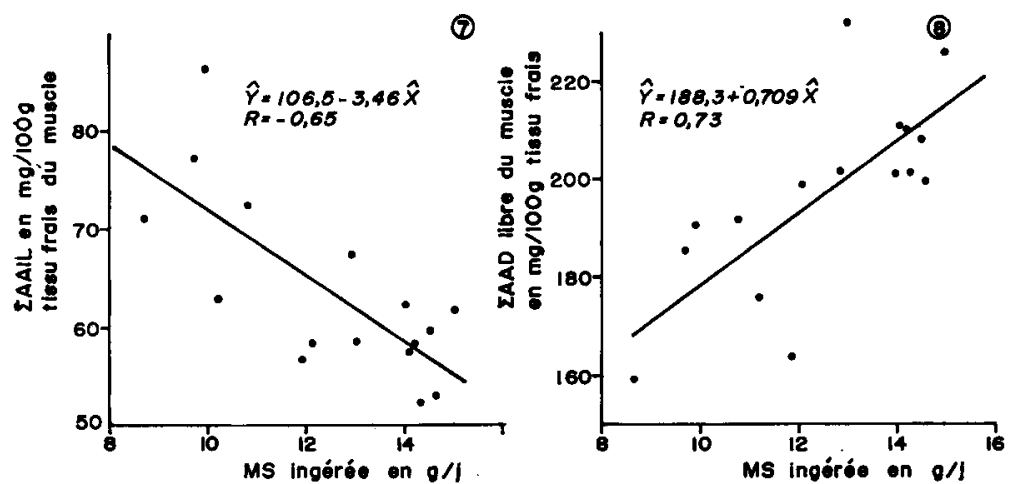

FIG. 7. - Somme des acides amines libres indispensables du muscle en fonction de la quantité de matière sèche ingérée

Fig. 8. - Somme des acides aminés libres non indispensables du muscle en fonction de la quantité de matière sèche ingérée

base de tourteau de tournesol, on n'observe pas de variations des teneurs en isoleucine libre du sang et du muscle. En revanche, DEAN et Scotr (I966) ont observé chez le poulet une augmentation de $192 \mathrm{p}$. Ioo de l'isoleucine libre du plasma lorsque l'apport alimentaire d'isoleucine passe de Ioo à I $50 \mathrm{p}$. Ioo du besoin et que les autres acides aminés sont apportés en quantités équilibrées. Il faut noter cependant que ces animaux reçoivent le régime expérimental pendant 6 heures par petits repas espacés toutes les demi-heures et que les acides aminés sont déterminés sur le plasma zo minutes après la dernière distribution d'aliment.

Nous n'avons pas, dans les conditions expérimentales utilisées (30 à r ro p. Ioo du besoin), constaté d'accumulation d'isoleucine libre dans le sang et le muscle du rat en croissance lorsque la teneur en isoleucine des régimes est légèrement supérieure au besoin : par contre, nous avons constaté que les teneurs en isoleucine libre des tissus étudiés sont faibles chez les animaux dont le régime contient des quantités d'isoleucine inférieures au besoin ; avec les régimes carencés en isoleucine, les croissances des rats sont peu élevées, ce qui peut être dâ, au moins en partie, à une diminution importante de la consommation d'aliment. Ce phénomène entraíne des carences multiples, notamment en énergie ; l'effet de celle-ci sur les teneurs en acides aminés libres indispensables n'est pas connu. Nous envisageons d'en entreprendre l'étude ultérieurerement.

\section{Reçu pour publication en mars 1971.}

\section{SUMMARY}

\section{EFFECT OF DIETARY ISOLEUCINE CONTENTS ON BLOOD} AND MUSCLE FREE AMINOACIDS IN THE GROWING RAT

I6 lots of growing rats were fed pure aminoacid diets ad lib. for $I_{4}$ to $I_{7}$ days. The diets were balanced for essential amino-acids except for isoleucine which was added at rates ranging from 36 to $\mathrm{r}$ Io $\mathrm{p}$. I oo of the requirement (Table I). Food intake, growth rate and free isoleucine contents of the blood and muscle (Table 2) were recorded.

With dietary isoleucin rates lower than the requirement, there was a drecrease in growth rate, food consumption and free isoleucine contents of the blood and muscle. With isoleucine rates higher than the requirement, no storage of isoleucine in blood or muscle was noticed under our experimental conditions. 


\section{RÉFÉRENCES BIBLIOGRAPHIQUES}

DeAN W. F., ScotT H. M., I 966 . Use of free amino acids concentration in blood plasma of chicks to detect deficiencies and excesses of dietary amino acids. J. Nutr., 88, 75-83.

Meister A. Biochemistry of the amino acids I965, vol. 2, p. 756-950 Academic Press, New York.

Pawlak M., Pron R., Ig68a. Influence de la supplémentation des protéines du blé par des doses croissantes de lysine sur la teneur en acides aminés libres du sang et du muscle du rat en croissance. Ann. Biol. anim. Bioch. Biophys., 8, 517-530.

Pawlar M., Pion R., I968 b. Influence de la supplémentation des protéines du blé par des doses croissantes de thréonine sur les teneurs en acides aminés libres du sang total et du muscle du rat en croissance. C. R. Acad. Sci. Paris, série D, 266, 1993-1995.

Pawlak M., PIon R, 1970. Utilisation des acides aminés du tournesol pour la croissance du rat; influence du taux protéique de la ration sur les teneurs en acides aminés libres du sang et du muscle. Ann. Biol. anim. Bioch. Biophys., 10, 3r7-322.

Ranhotra G. S., Connor-Johnson B., 1965. Effect of feeding different amino acid diets on Growth Rate and nitrogen retention of weanling rats. Proc. Soc. Exp. Biol. Med., 118, I 197-1201.

Richardson L. R., Cannon Martha L., WebB B. D., r965. Relation of dietary protein and lysin to free amino acids in chicks tissues, Poultry Sci., 44, 248-257. 